NOTA

297-310

HACIA LA ENSEÑANZA DE LOS ESTUDIOS ARTÍSTICOS EN CHILE: MANUEL DE SALAS Y LA ACADEMIA DE SAN LUIS ${ }^{1}$

Toward the education of artistic studies in Chile: Manuel de Salas and the St. Louis Academy

Noemi Cinelli*

A Fedele de Luca, in memoriam

\title{
ESTADO DE LA CUESTIÓN
}

El artículo que presentamos trata de la situación de la enseñanza de las Bellas Artes en Chile. Particular atención será dedicada a las etapas fundamentales de la fundación de la santiaguina Academia de San Luis, en el delicado momento de transición entre la época colonial y el proceso de autodeterminación de Chile que llevaría a la proclamación de la Independencia en 1818 (Ramírez Rivera; Hancock; Villalobos).

El motivo de la elección del tema radica en la voluntad de quien escribe de reconocer merecido prestigio al ilustrado chileno don Manuel de Salas en el impulso dado a las Bellas Artes, mediante su primera tentativa de implantación de los estudios de dibujo en el país a la sombra de los Andes (Amunátegui, Apuntes; Celis).

Es nuestra convicción que su aportación va mucho más allá de la actividad desarrollada en la Academia de San Luis, es decir, que a Salas se debe el nacimiento del primer debate científico respecto de la educación artística en Chile.

Hasta ahora los estudios de temas chilenos dieciochescos han sido escasos, en especial lo referido a nuestro objeto de estudio, frente, por ejemplo, al interés despertado por los grandes retratistas de la época inmediatamente sucesiva a la que aquí tomamos en consideración.

Creemos que este hecho se deba al afán de los investigadores empeñados en temas chilenos de indagar acerca de las urgentes cuestiones no solo culturales, sino más bien políticas y sociales procedentes de la proclamación de la República, con el objetivo común de dar forma a una historia del País y por tanto de una identidad nacional.

A esto debemos añadir la constatación de la indiscutible dificultad de hallar escritos sobre la época, por un lado a causa de las pérdidas y las dispersiones que registran las instituciones entre sus fondos y por el otro por las continuas incursiones de los coleccionistas privados en el mundo de las fuentes documentales (Vergara, 14-16).

\footnotetext{
${ }^{1}$ Este artículo ha sido elaborado en el marco del proyecto Postdoctorado Fondecyt 2014 No3140109: "Poder y representación en la América colonial: el retrato de las élites en Chile en el siglo XVIII", del que la autora es Investigadora Responsable.
} 
La Situación DE las Bellas ARTeS en ChILE en el Siglo XVIII: ALGUNaS CONSIDERACIONES

Dejando de lado en esta sede las consideraciones acerca del contexto histórico chileno, para cuya reflexión recopilamos la bibliografía más reciente al final de este trabajo, creemos que para arrojar luz diferente respecto de los alcances de la propuesta de don Manuel de Salas sean necesarias algunas precisiones acerca del panorama cultural y educativo chileno a finales del siglo XVIII.

La coyuntura económica en la que Chile se encontraba después de las reformas propias de la modernización borbónica, hacía que la educación y las actividades culturales en particular no fuesen una de las prerrogativas que más preocupaban e interesaban a la sociedad, derivando ello en la ausencia de un organismo central y secular que tutelara las tareas relacionadas con la enseñanza.

Además, en 1767 por orden de Carlos III de Borbón los jesuitas fueron expulsados también del territorio chileno ${ }^{2}$, quedando el país, que progresaba culturalmente con el perseverante fomento de la Iglesia, en una fase de retraso en este ámbito (Enrich).

En efecto, a los jesuitas además de la fundación de escuelas de primeras letras y del desarrollo de archivos y bibliotecas, se debía el sistema de enseñanza más eficiente y completo que tuvo Hispanoamérica entre los siglos XVI y XVIII y que además abarcaba los tres niveles de la enseñanza: básica, media y terciaria. Con el fatídico 1767 se interrumpió el avance de las formas básicas de institucionalidad cultural en Chile (Vergara, 63) ${ }^{3}$.

Otro aspecto a subrayar se refiere al rol jugado por Perú, centro de producción cultural muy activo en la época que nos interesa, desde cuyos territorios se propagaban los conocimientos en el área andina, y que en cuestiones de Bellas Artes, además de las técnicas de pintura, señalaba las directrices a seguir en materia de gusto y temáticas (De Mesa y Gisbert).

En Chile la vida cultural tenía su centro neurálgico, como es de esperar, en Santiago, donde se encontraban las grandes haciendas del país. El desarrollo económico de la capital no tenía correspondencia desafortunadamente con el avance en las actividades en torno a la literatura, las Bellas Artes, y la música que quedaban limitadas a una restringida élite del patriciado santiaguino.

Para poder comprender aún mejor la situación, es necesaria otra precisión: la separación entre las ideas que circulaban en estas pequeñas élites y la práctica artística de la época. En efecto, las ideas que en esta sede por comodidad definimos

\footnotetext{
${ }^{2}$ El papa Clemente XIV Ganganelli firmó la extinción canóniga de la Compañía de Jesús en 1773 con la Bula Dominus ac Redemptor.

${ }^{3}$ Por contra este hecho hizo que las ideas de Carlos III acerca de la educación encontraran terreno fértil para el desarrollo de formas de enseñanzas seculares.
} 
neoclásicas ${ }^{4}$, perfectas expresiones en Bellas Artes de los ideales de la Revolución del Siglo de las Luces, eran las que informaban del debate acerca del arte. De todas formas, estas no podían encontrar rápida actuación en la práctica, ya que el repentino alejamiento de las ideas de la Iglesia en lo referido a temas tratados y mecenazgo artístico, podía considerarse una insubordinación al orden dominante ${ }^{5}$.

El rol del Convictorio Carolino, el Colegio Máximo de San Miguel, y otros colegios de primeras letras puede ayudarnos en la comprensión de la historia de la educación en Chile, aunque la escasa literatura sobre estas instituciones es un obstáculo al conocimiento del desarrollo de los cursos de arte y dibujo (Frontaura y Arana) ${ }^{6}$.

UNA ÚlTIMA CONSIDERACIÓN ANTES DE DEDICARNOS A LA FIGURA DE MANUEL DE SALAS

Hasta 1652 no encontramos noticias acerca de formas de enseñanza artística en Chile. En esta fecha Francisco de Escobar, Damián Muñoz y Crisóstomo Atahualpa se dedicaban a la formación de sus discípulos mediante el estudio de tres tipos de pintura: la enseñanza desarrollada en Cuzco que tenía como objeto ejercicios pequeños en los que quedaban patentes ciertas influencias flamenca y bávara (Hempe); la pintura de retrato que tuvo poco tratamiento y difusión; y por último la pintura a la romana, con el paisaje como protagonista y el dibujo como base, realizada mirando constantemente al Renacimiento europeo como fuente de inspiración. Aunque prometedoras, estas formas de enseñanza nunca tuvieron una sistematización ni una reglamentación.

Habría que esperar a don Manuel de Salas para que en Chile cambiara la idea de dibujo, su aplicación, su enseñanza.

\section{DON MANUEL DE SALAS: UN ACERCAMIENTO A SU FIGURA}

Manuel Silverio Antonio de Salas y Corbalán, esto su nombre completo, nació en Santiago el 19 de junio de 1754, donde murió el 28 de noviembre de 1841. Su familia formaba parte de la más distinguida aristocracia andina (Amunátegui, Don Manuel).

\footnotetext{
${ }^{4}$ Creemos que el libro de Mario Praz Gusto neoclassico, es clave para entender el significado del término neoclásico, que no quiere definir un estilo, sino más bien una cultura figurativa de la segunda mitad del siglo XVIII.

${ }^{5} \mathrm{~A}$ tal propósito basta con citar el episodio del destierro de un grupo de intelectuales chilenos que se opusieron a las ideas de la Corona, y que en noviembre de 1824 fueron exiliados en la isla Juan Fernández. ${ }^{6}$ El Colegio de Naturales de Chillán estaba administrado por los franciscanos, pero resultó tener muy poco éxito si consideramos que en 1790 contaba con menos de 15 alumnos. El Convictorio Carolino, llamado así en homenaje a Carlos III de Borbón, ejercía como una Escuela. El Colegio Máximo de San Miguel fundado en el siglo XVII fue transformado en Universidad en 1625, aunque nunca actuó como tal.
} 
Desde muy joven desempeñó varios cargos públicos, entre ellos, fue regidor del Cabildo de Santiago en 1775, y en 1796 diputado del barrio de la Catedral con cinco cuadras de jurisdicción.

Después de una etapa en Europa, concretamente en España donde viajó a Madrid y Cádiz debido a los encargos políticos de su padre entre 1777 y 1782, es evidente la influencia que sobre Salas ejercieron las lecturas de los grandes teóricos de la Ilustración, derivando ello en el acercamiento cada vez más fuerte a la causa de la Independencia del País, que años más tardes le valió una época de exilio en la Isla Juan Fernández.

Salas fue parte activa en la elaboración del Reglamento Constitucional en 1812, después haberse garantizado el año anterior un sitio en el Olimpo de los humanistas gracias a la promulgación de la Ley de Libertad de Vientres, que ordenaba el término de la esclavitud para los hijos de esclavos nacidos en territorio chileno, prohibiendo además el ingreso de nuevos esclavos a Chile?

Para comprender mejor la personalidad de Salas, sus intereses y sus influencias políticas y morales, podemos acudir a una carta que el 25 de enero de 1773 dirigió desde Lima a José Antonio Rojas:

Estimaré a V. que agregue a mis encargos una flauta buena i algunas estampas tanto de humo como a buril, por que estoi tomando lecciones de dibujo; i para este mismo efecto envíeme V. a Palomino, 2 tomos; a Samuel Marolois, "De arquitectura y perspectiva" 2 tomos; a Lebrun, "La Historia de Alejandro" i la de "Don Quijote", estampadas; a Gerardo Lairesse, "Principios de Dibujos", un tomo en folio; a Don José de Ribera, alias El Españoleto. Añada V. a estos autores algunos colores que acá no se hallan, como son, alcorca de grano, carmín superfino de Florencia y azul ultramar. Mis hermanos me dicen le pida a V. "los Viajes de Ciro" en francés i "Mil y un Día". Para mí envíe "El Paraíso Perdido" de Milton; las "Poesías del Abate Grecourt", "El Espirit", "El Filósofo Sans Souci", "El Belisario", y unas "Memorias sobre América y los Americanos", que nuevamente han salido en francés, anónimas en tres tomos [...]. Renuevo a V., aunque parezca majadería, todos mis encargos. Me da motivo para esto, la satisfacción que de V. tengo i la escasez que hay por acá de todas las cosas, tanto que hasta las putas se han acabado; y así cuando $\mathrm{V}$. venga, traiga cien cajones de ellas, que tendrán pronta salida ${ }^{8}$.

Pereira Salas (177) en su escrito subraya que la obra de Palomino -que De Salas encarga- es su obra maestra, esto es, El Museo Pictórico y Escala Óptica de 1715 , es un tratado que sin duda ejerció una influencia determinante en la elaboración de las teorías de De Salas. Las actuaciones del santiaguino en efecto

\footnotetext{
${ }^{7}$ En 1823 De Salas promovió la total abolición de la esclavitud en Chile.

${ }^{8}$ Transcribimos parte de la Carta de Manuel de Salas a José Antonio Rojas, 25 de enero de 1773, con la misma ortografía con la que se publicó en el tercer y último tomo de Escritos de Don Manuel Salas relativos a él y su familia, p. 116, editado por la Universidad de Chile en 1914.
} 
están completamente en línea con las ideas de Palomino, especialmente en la vinculación entre geometría y dibujo.

Entre otros autores encontramos mención a Samuel Morolois, Mathematicum opus absolutissimum: continens geometriae, fortificationis, arquitecturae \& Perspectivae Teoriticae ac practicae regulas, demonstrationes, \& figuras perfectissimas, obra de 1638, que trata el tema de los problemas de medidas, proporciones, perspectivas y vistas.

Charles LeBrun y sus estampas acerca de la vida de Alejandro Magno son una lectura muy interesante por su vinculación con la academia de pintura, cuyas ideas ya circulaban entre los intelectuales chilenos.

En la citada carta a Rojas, De Salas pide el envío del manual de dibujo de Gerard de Lairesse, dibujante y grabador valón, Grondlegginge der Teekenkunst zynde een korte en zeekere weg om door middel van de geometrie of meetkunde, de teeken-konst volkomen te leeren, cuya mención en el listado de libros solicitados es interesante, ya que propone ejercicios de copia como método de aprendizaje, proporciona consejos del maestro, afronta de manera científica la teoría del arte que es estructurada didácticamente, tal y como De Salas preveía.

Sorprende encontrar el Paraíso Perdido de John Milton, de quien ya unos años antes (en 1644) habían sido publicadas sus reflexiones Sobre la Educación. Creemos que la predilección de De Salas por este autor se deba más a este escrito que a su obra maestra. En su especulación Milton relaciona aspectos propios de la religiosidad y de la ética con los aspectos positivos de la educación clásica aplicados a un ciudadano para el mejoramiento de su país.

Los escritos firmados por De Salas prueban su competencia en materias científicas y tecnológicas, su manejo de los procesos propios de la industria minera, individuando en este recurso del que Chile abundaba la rentabilidad económica que podría derivar de una explotación sistemática y racional.

En el Memorial de 1801, escrito cuando desempeñaba el cargo de Síndico del Consulado, queda evidente el entendimiento ecléctico y completo del concepto de educación, más allá de la enseñanza. Encontramos una referencia a la obra del Conde de Campomanes, Discurso sobre la educación popular de los artesanos y su fomento de 1755:

Nuestra España, siempre fecunda de grandes hombres, solo espera para producirlos que se les presente ocasión de distinguirse. Poetas guerreros ilustres, políticos consumados, teólogos insignes, todo lo ha tendido cuando las circunstancias lo han requerido, y más que todo, cuando los dispensadores del honor les han dirigido nuestras miradas benignas. [...] huyen cubriéndose los ojos, el interés particular, la indecisión, al amor a los errores antiguos, el error a las verdades nuevas; y sustituyéndose en lugar del fatal egoísmo aquel espíritu público que solo halla morada en las almas virtuosas y grandes, se limpia el horizonte, y su libro de oro, La Industria y Educación Popular, se lee con entusiasmo (De Salas, Escritos, 215-216). 
El Conde de Campomanes (Pedro Rodríguez), cuyo pensamiento económico le valió la palma de representante más logrado del despotismo ilustrado de la época de los Borbones, fue partidario de la idea del libre ejercicio de la artesanía fuera de los circuitos de las cofradías y de los gremios, que en su opinión solo limitaban el trabajo del individuo.

El asturiano, en quien De Salas se inspiró claramente, expuso sus ideas acerca de las leyes para reglamentar las artes recurriendo a palabras como: orden público, ley natural y educación; legitimó la subordinación y la necesidad de disciplina de los artesanos, determinó la justa práctica en sus oficios, descalificando los saberes locales de los artesanos y planteando su regulación por medio de la sistematización de su educación:

En los gremios de artesanos hay poquísima enseñanza. Falta dibujo en los aprendices, escuela pública de cada oficio y premios de cada oficio y premios a los que adelanten o mejoren la profesión. Todo es tradicionario y de poco primor en los oficios por lo común (Rodríguez, 18)

Utilizando un lenguaje menos culto de Campomanes y más asequible para las élites santiaguinas, De Salas elaboró un texto fundamental para entender su compromiso con la educación. Nos referimos al Reglamento del Gremio de los Plateros, fechado al 9 de abril de 1802, en el que argumentaba entre otros asuntos acerca de la íntima relación que une maestro y discípulo en el proceso de aprendizaje, acerca de la formación de los artesanos:

$8^{\circ}$ Durante este tiempo, será obligación del maestro enseñarle el oficio y la doctrina cristiana, darle buen ejemplo, procurar que aprenda á leer, escribir, la aritmética vulgar y el dibujo, como requisitos todos, sin los que jamás podrá poner tienda pública. Deberá vestirlo modestamente, darle alimentos, y cama proporcionada a un muchacho pobre. Si al entrar el aprendiz trae vestuario y cama, se le señalará menos tiempo que al que sea necesario costearle uno y otro, al árbitro del Juez, para que la mayor duración del servicio compense el gasto. Podrá ser corregido de sus faltas o desaplicación, pero del modo que lo sería un hijo, y sin usar jamás del afrentoso azote, que solo sirve para envilecerlos y prostituirlos (De Salas, Reglamento, en línea).

La idea de aprendizaje está íntimamente relacionada con el concepto de maestro, una guía que orienta al aprendiz y que se ocupaba de tres materias a la vez.

Todo ello forjó en De Salas una idea de enseñanza que respondía a tres principios de los que nunca se alejaría: todas las disciplinas tienen igual importancia y no existe una diferenciación entre las principales y las secundarias; la enseñanza del dibujo conlleva el aprendizaje con modelos vivos; las clases del dibujo requieren necesariamente ser dirigidas por maestros que sigan un plan de estudio establecido.

\footnotetext{
9 El texto completo puede consultarse en la Biblioteca Nacional de Chile y en: http://archive.org/stream/donmanueldesalas01amun/donmanueldesalas01amun_djvu.txt
} 
De Salas determina así una correspondencia entre arte y dibujo que hasta aquel entonces no se había tomado en consideración en el panorama de las Bellas Artes chilenas.

\section{LA ACADEMIA DE SAN LUIS EN EL PROYECTO DE DE SALAS}

La primera referencia a la idea de poner en marcha una academia en Santiago data del 1 de diciembre de 1795, en el documento que De Salas dirigió a los Señores de la Junta del Consulado de Chile (Berríos, Del taller, 38). La relevancia de tal escrito titulado: Representación al consulado sobre la necesidad de establecer un aula de matemáticas radica en diferentes cuestiones que levantó su autor:

- El dibujo es una disciplina separada de la geometría y de la aritmética, pero al igual que estas dos últimas, es materia indispensable para poder desenvolverse con maestría tanto en la agricultura como en el comercio y la industria.

- Para que florezcan las Bellas Artes en Chile es esencial que los artistas dominen los principios del dibujo, a falta de lo cual, las artes no pueden hacer el menor progreso.

Estas ideas acerca del dibujo dejan de manifiesto cuánto De Salas se había embebido de las ideas de la Ilustración que circulaban en Europa. En este sentido Sol Serrano subraya que en Chile los vientos de reforma que soplaban en época borbónica fueron recibidos positivamente por los criollos, siendo De Salas su representante más ilustre (Serrano, 249). En las palabras de Isabel Cruz de Amenábar se debe al "destacado papel en el desarrollo cultural de las postrimerías de la Colonia [...] ilustre filántropo, educador, promotor de las artes e industrias diversas, quien representa acabadamente la influencia de la mentalidad ilustrada en el Chile de fines del siglo XVIII" (99).

En la Academia confluyeron, en efecto, la huella del Siglo de las Luces, y la voluntad de Manuel de no romper drásticamente con la tradición chilena sino que quiso impulsar el progreso nacional mediante la asimilación paulatina de la lección enciclopedista del pragmatismo y utilitarismo.

En este sentido queda patente la influencia francesa que impregnó de manera esencial el ámbito educacional chileno y que habría llevado a la fundación de la Universidad de Chile algunos años más tarde, de los que tomamos en consideración en nuestro escrito.

Siguiendo con la carta de 1795, aunque su autor no nos proponga argumentos científicos en algunos puntos claves, como el de la necesidad de estudiar los principios de la física, de la metalurgia, la mineralogía, para que Chile pueda explotar racionalmente sus recursos mineros de los cuales abundaba, debemos subrayar la intrépida voluntad que De Salas manifiesta a la hora de proponer una solución:

No debiendo dudarse de la necesidad que hai de tener principios de jeometría, aritmética i dibujo, para tratar con acierto de la agricultura, comercio é industria. 
Parece que de ningún modo pueden VV.SS. llenar mejor el encargo de proteger i promover estos tres ramos que proporcionando á la juventud tales conocimientos. [...] Lo conseguirán destinando la sala inmediata al tribunal que durante el día sirve de recibimiento ó antesala, para que en ella oigan al principio de la noche, lecciones de estas tres partes de las matemáticas los alumnos que quieran, sin haber más gasto que el de papel (De Salas, Escritos Vol. I, 567).

El santiaguino era consciente de los problemas financieros que el Consulado habría llevado a colación para dar una negativa a su solicitud, así que a la hora de dirigirle la carta, en ella presentaba también la posible organización de la institución: las clases se dictarían en la antesala del tribunal en horarios vespertino, el maestro había sido individuado en la persona del arquitecto reconocido por las academias de Roma y por la Academia de San Fernando de Madrid, Joaquín Toesca, y los modelos para copiar los habría puestos a disposición De Salas recurriendo a su propia colección.

Por las palabras de De Salas quedaba claro que el único gasto del que debería encargarse el Consulado era el papel.

Un mes después de enviar la Representación, precisamente el 12 de enero de 1796, De Salas recibió la respuesta del Consulado, que denegaba la posibilidad de abrir la Academia con las siguientes palabras:

Vista la representación del Síndico sobre que da cuenta del Consulado, se fomente la instrucción de los jóvenes en las artes i en las matemáticas, geometría, aritmética i dibujo. Precisas para tratar con acierto de la agricultura, comercio é Industria, en la Junta de Gobierno resolvieron los SS. que la componen, que por ahora es inadaptable el proyecto aunque laudable por los objetos de pública utilidad que embebe en sí con respeto á que los fondos del Consulado aun no sufragan para las moderada dotación de sus empleados (De Salas, Escritos, Vol. I, 569).

Sin darse por vencido De Salas retomó la pluma este mismo día rogando que revisaran la decisión tomada: sus esfuerzos se vieron premiados, ya que el 24 de julio de 1796 Carlos IV firmó en su residencia de Aranjuez una Real Orden que pedía la creación de una Escuela de Aritmética, Geometría y Dibujo ${ }^{10}$, sin que recibiera aún la existencia legal. Para ello De Salas debería esperar el 31 de enero de $1798^{11}$, fecha del decreto que la nombraba San Luis en homenaje a reina de España muerta precozmente.

De Salas no se desanimó, y en marzo de 1797, haciendo recurso al teniente general don Gabriel de Avilés, se ordenaba la apertura de la Academia de San Luis, con la protección de este último gobernador del Reino de Chile, teniendo su sede en la actual calle San Antonio. La guerra entre España e Inglaterra fue la causa de que

\footnotetext{
${ }^{10}$ Archivo Nacional de Chile, Fondo Varios, vol. 754, s.n.

${ }^{11}$ Archivo Nacional de Chile, Fondo Varios, vol. 747, s.n. 
algunos fondos no se recibieran, de este modo, Manuel de Salas tuvo que aportar dinero de su propio sueldo. Ya algunos meses antes de recibir el decreto de 1798, es decir en septiembre de 1797, la Academia de San Luis empezó a funcionar ${ }^{12}$ contando tan solo con el apoyo del Ayuntamiento y del gremio de los Comerciantes.

En efecto, y como era de prever, no faltaron problemas de diferente índole a la hora de empezar las clases, y la primera dificultad con la que De Salas tuvo que enfrentarse fue exactamente la falta de recursos disponibles.

No obstante la orden del rey que establecía que el dinero tenía que proceder desde el Consulado y del Tribunal de Minería, este último siempre demostró cierta animadversión para los planes en De Salas, decidiendo no proporcionar el dinero que debía hasta que recibió la orden real que establecía los 1.000 pesos al año a versar obligatoriamente (Amunátegui y Solar, 16).

Las clases de dibujo empezaron a dictarse como previsto en la noche, y contaban, como se ha señalado, con instrumentos pertenecientes al mismo De Salas quien puso a disposición cien modelos, mesas, bancos, candeleros, como él mismo informa en la Representación. La feliz casualidad de la presencia temporánea del profesor italiano Martín Petri en Chile hizo que pudiese encargarse durante un año y medio de las clases citadas, mientras que el mencionado arquitecto Toesca, también italiano, dictaba las de geometría y aritmética.

Cuando Petri tuvo que seguir con su viaje a Perú en 1798, su cargo fue tomado por Ignacio Fernández Arrabal, procedente de la Casa de la Moneda, donde ejercía como platero, y que ya conocía la Academia de San Luis por haber sido uno de sus alumnos.

Con la Real Orden del 7 de junio de 1801, que determinaba la suspensión del pago de los 1.000 pesos del Tribunal de Minería, la Academia ${ }^{13}$ vio sus cuentas refutadas, quedando De Salas en la condición de tener que cerrar las puertas de la institución.

Una vez más, con mucha tenacidad, decidió dirigirse directamente al gobernador de Chile Luis Muñoz de Guzmán, con una carta fechada el 12 de agosto de 1802, pidiendo que se discutiera otra vez la cuestión de la subvención del Tribunal:

La voluntad del Rey, no es extinguir una Escuela, a cuyo favor ha expedido siete ordenes reales $[\ldots]$ Lo que se manda es únicamente suspender sus rentas por ahora, esto es, mientras subsisten las causas que representó el Tribunal de Minería. Siendo pues este el término de la Real Orden, siendo de tan malas consecuencias cualquiera otra inteligencia que se le dé, y no resultando inconveniente alguno de concebirla así, no dudo que convendrán en su permanencia los mismos cuerpos á quien debe el $\operatorname{ser}^{14}$.

\footnotetext{
${ }^{12}$ Hay informaciones contrastantes acerca del día preciso de comienzo de las actividades. Según Eugenio Pereira Salas fue el 8 de septiembre, mientras que Amunátegui hace empezar las clases el día 18 del mismo mes (Pereira, 179; Amunátegui y Solar, 18).

${ }^{13}$ Archivo Nacional de Chile, Fondo Varios, Vol. 749, No II.

${ }^{14}$ Recopilamos manteniendo la sintaxis original de las palabras de la Carta de Manuel de Salas enviada al Gobernador de Chile Luis Muños de Guzmán, 12 de agosto de 1802, Archivo Nacional de Chile, Fondo Varios, Vol. 155, s/n.
} 
Las palabras de Manuel de Salas dieron sus frutos y la Academia pudo seguir con sus cursos, además de seguir otorgando incentivos pecuniarios a los mejores alumnos del curso de matemática.

El 18 de agosto de 1805 se emitió una orden que anulaba la decisión de 1801, cuya oficialidad llegaba a Chile en el año $1806^{15}$, naciendo así la Academia. No obstante, Manuel de Salas ya llevaba nueve años trabajando por el funcionamiento de la institución y el progreso de sus alumnos.

La Biblioteca Nacional de Santiago guarda el Libro de cuentas de la Academia. El documento nos proporciona interesantes noticias acerca del plan docente, desarrollado de acorde a cuatro cátedras, Dibujo, Matemática, Gramática, Primeras Letras. No obstante las continuas referencias a la creación de una cátedra de mineralogía y de química, el Libro no deja dudas: nunca se implantaron estas disciplinas, ya que no hay constancia de profesores pagados por ello (Amunátegui, Los primeros años, 45-57).

Otra información interesante se refiere al profesor que cobraba más, que era el de dibujo, con 400 pesos anuales, quedando claro el prestigio y la importancia que se le reconocía dentro del organigrama de la institución.

\section{EL OCASO DE LA ACADEMIA DE SAN LUIS}

El destino de la Academia después reunida la primera Junta Nacional de Gobierno el 18 de septiembre de 1810 no podría seguir siendo el de una institución que gozaba de apoyo de la realeza, así que De Salas, muy astutamente, dejó manifiesta la exigencia de incorporar la Academia al ya citado Convictorio Carolino. En el escrito del ilustrado fechado al 11 de abril de 1811, dirigido al Presidente y a los Vocales de la Junta de Gobierno, tuvo cabida también el deseo de ver otra vez en marcha el curso de dibujo que llevaba sin dictarse desde diciembre de 1799 por falta de profesores que pudiesen encargarse de ello:

Llenaría todas estas miras y deseos del público el establecimiento de un colegio en que se enseñasen los principios de toda buena educación, esto es la primeras letras, idioma, dibujo, moral, aritmética, geometría y demás que sirven á formar el juicio y a preparar para estudios más serios (De Salas, Escritos Vol. I, 631) ${ }^{16}$.

Aunque la Junta no satisfizo la solicitud de Manuel de Salas, hay que subrayar que contemporáneamente se asistió en Chile al establecimiento de diferentes instituciones que se preocupaban de la educación pública y privada.

La Comisión de Educación que se creó en esta nueva etapa histórica dispuso la fundación de un único centro de educación estatal que fusionara la Academia de San

\footnotetext{
${ }^{15}$ Archivo Nacional de Chile, Fondo Varios, Vol. 254, s.n.

${ }^{16}$ Recopilamos parte de la "Carta de Manuel de Salas, del 20 de febrero de 1811, dirigida al Presidente y a los vocales de la Junta Provisional de Gobierno", sin modificar la ortografía original.
} 
Luis, la Universidad de San Felipe y otros colegios, primera tentativa del gobierno recién formado de controlar la educación en Chile. En el documento que se generó para sancionar dicha decisión leemos lo siguiente:

Como la Universidad queda incorporada al Instituto, del modo prevenido en el prólogo será en adelante la academia de los sabios y el museo de las ciencias de que serán miembros los doctores, maestros y bachilleres recibido hasta la instalación de esta constitución; pero en adelante solo obtendrán estos grados los que en cada profesión hayan observado el tiempo y estudio que se señala la misma [...] Se une a la Universidad, la Sociedad Filantrópica, la Academia de Práctica, bajo sus respectivos reglamentos. [...] En las diversas salas de la misma Universidad se deberán situar el Gabinete de historia natural para lo que se trasladará allí el principio colectado que existe en la Academia, con sus correspondientes estantes. [...] Su custodia y primera clasificación correrá al cargo de los catedráticos de Chímica, Botánica, y Física, bajo la inmediata inspección del virtuoso ciudadano don Manuel de Salas promotor originario de este establecimiento ${ }^{17}$.

La estructura del nuevo aparato pedagógico que confluyó en el Instituto Nacional y que establecía el estudio de materias como la geometría y el dibujo junto con la aritmética y la botánica, se debe claramente al modelo usado en la Academia de San Luis por Manuel de Salas. Gracias a la lectura del informe del 10 de abril de 1801 conocemos el modelo en que hacía años se había inspirado De Salas para promover la institución. Estas eran las academias de las que durante su viaje a España y gracias a sus lecturas se había podido informar adecuadamente, es decir, la San Fernando de Madrid, la de Sevilla y de Málaga.

En los años que siguieron a la fusión de los diferentes centros educacionales, se trabajó para dar vida propia e identidad al Instituto; a ello contribuyeron destacadas personalidades de la élite cultural chilena, entre ellos es importante citar a Juan Egaña y Francisco de Echaurren que en 1823 firmaron el informe que conllevó a la fundación del Instituto un mes después. De Salas también dio su contribución a ello, hasta que el viaje que tuvo que emprender a Uruguay le alejó de Chile.

En el proyecto firmado por el citado Egaña, el Instituto debía albergar en su sede el Anfiteatro Anatómico, la Academia de Leyes y Prácticas Forenses, un Gimnasio, un Jardín Botánico, una Escuela de Pintura y Escultura, el Gabinete de Historia Natural, el Laboratorio Químico y la Escuela del Dibujo. Estos últimos eran los centros que De Salas había previsto implantar en la Academia de San Luis para la formación de los jóvenes que se preparaban para el mundo del arte y de la industria, aunque en la realidad solo consiguió implantar la cátedra de dibujo.

\footnotetext{
17 "Decreto de la Junta Nacional de Gobierno que incorpora la Universidad de San Felipe al Instituto Nacional. Firmado en el Palacio de Gobierno: Francisco Antonio Pérez, Agustín de Ayzaguirre, Juan de Egaña, José Tadeo Mancheño". Santiago de Chile, 2 de agosto de 1813.
} 
Egaña estaba poniendo las bases de la futura Escuela de Bellas Artes en Chile gracias a lo empezado por Manuel de Salas.

Citando las palabras de Eugenio Pereira Salas: "la influencia de la Academia en sus diecisiete años de actividad fue poderosísima, sembró la semilla de la enseñanza científica y sus alumnos pasaron a ser los primeros alarifes y agrimensores con preparación técnica y profesión" (183).

Para monitorear el desarrollo de la enseñanza del dibujo en Chile, podemos referirnos a un informe de 1813 acerca de la organización del Instituto Nacional: este se orientaba a un ejercicio práctico más cercano al ámbito de las Bellas Artes, en particular al retrato y al paisaje (Amunátegui y Solar, 160-168).

Se estaba paulatinamente produciendo un cambio importante: si Manuel de Salas había introducido el discurso del dibujo dentro de una perspectiva técnica y científica más que propiamente pertinente al ámbito de las Bellas Artes, el nuevo impulso dado por Egaña lo estaba dirigiendo hacía la formación de jóvenes artistas.

Como bien subraya Sol Serrano, la Academia de San Luis permanece como la primera experiencia educacional chilena de clara procedencia e inspiración ilustrada, y que resume las dos promesas que la próxima Independencia realizará: la confianza en el pensamiento científico como instrumento para transformar positivamente la realidad; y una mayor presencia del Estado en el fomento de este incontenible cambio (Serrano, 251).

Fue en definitiva un centro técnico y pragmático, que tenemos que considerar como el legajo intelectual de más impacto que De Salas consignó a la sociedad chilena, el fruto de su mente ilustrada, y resultado de sus esfuerzos incansables. No al azar el día de la muerte de Manuel de Salas, el 28 de noviembre de 1841, se declaró duelo nacional.

\author{
Universidad Autónoma de Chile* \\ Mapocho 1450, piso 413, Santiago (Chile) \\ noemicinelli@gmail.com
}

\title{
OBRAS CITADAS
}

AA.VV. De la etnohistoria a la historia en los Andes: $51^{\circ}$ Congreso Internacional de Americanistas celebrado en Santiago de Chile en el año 2003. Quito: Abya Yala, 2008.

AA.VV. La misión y los jesuitas en la América española, 1566-1767: cambios y permanencias. Madrid: Consejo Superior de Investigaciones Científicas, 2005.

AA.VV. Historia de Chile, 1808-1994. Cambridge: Cambridge University Press, 1998. Amunátegui, Miguel Luis. Don Manuel de Salas. 1754-1841. Santiago de Chile: Imprenta Nacional, 1895. 
_- "Apuntes sobre lo que han sido las Bellas Artes en Chile". Revista de Santiago, $\mathrm{N}^{\circ}$ II (abril 1849): 37-47.

Amunátegui y Solar, Domingo. Los primeros años del Instituto Nacional. Santiago de Chile: Imprenta Cervantes, 1889.

Archivo Nacional de Chile, Fondos Varios, Vol. 254/ Vol. 747/ Vol. 749/ Vol. 754/ Vol. 801.

Berríos, Pablo; Cancino, Eva, et al. Del taller a las aulas. La institución moderna del arte en Chile (1793- 1910). Santiago de Chile: LOM Ediciones, 2009.

Celis, Luis. Manuel de Salas. Pensamiento educativo en tiempo de transición. Revista pensamiento Educativo 34, 1, (junio 2004): 18-27.

Cruz de Amenábar, Isabel. Arte: lo mejor en la historia de la pintura y escultura en Chile. Santiago de Chile: Editorial Antártica, 1989.

Decreto de la Junta Nacional de Gobierno que incorpora la Universidad de San Felipe al Instituto Nacional. Firmado en el Palacio de Gobierno: Francisco Antonio Pérez, Agustín de Ayzaguirre, Juan de Egaña, José Tadeo Mancheño, Santiago de Chile, 2 de agosto de 1813.

De Mesa, José y Gisbert, Teresa. Historia de la pintura cuzqueña. Lima: Fundación Agusto Wiese, 1982.

De Salas, Manuel. Escritos de Don Manuel de Salas y documentos relativos a él y su familia. Santiago de Chile: Universidad de Chile, 1914, Vol. I, II, III.

_ Carta de Manuel de Salas a José Antonio Rojas, 25 de enero de 1773. En Salas Lavaqui, Manuel. Escritos de Don Manuel de Salas relativos a él y su familia, Tomo II. Santiago de Chile: Imprenta Cervantes, 1910.

— Reglamento del Gremio de los Plateros. Santiago de Chile: 1802. Disponible en: http://archive.org/stream/donmanueldesalas01amun/donmanueldesalas01amun djvu.txt

— Carta de Manuel de Salas enviada al Gobernador (Presidente) de Chile Luis Muños de Guzmán, 12 de agosto de 1802. Archivo Nacional de Chile, Fondo Varios, Vol. 155.

_- Carta de Manuel de Salas, del 20 de febrero de 1811, dirigida al Presidente y a los vocales de la Junta Provisional de Gobierno. Archivo Nacional de Chile, Fondo Varios, Vol. 155.

Enrich, Francisco. Historia de la Compañia de Jesús en Chile. Barcelona: Imprenta de Francisco Rosal, 1891.

Frontaura y Arana, José Manuel. Historia del Convictorio Carolino. Santiago de Chile: Imprenta Nacional, 1889.

Hancock, Anson Uriel. Historia de Chile. Madrid: La España Moderna, 1893.

Hempe Martínez, Teodoro. La tradición clásica en el Perú Virreinal. Lima: UNMSM, Fondo Editorial, 1999.

Pereira Salas, Eugenio. Historia del Arte del Reino de Chile. Santiago de Chile: Ediciones Universidad de Chile, 1965. 
Praz, Mario. Gusto Neoclassico. Milano: Rizzoli, 1974.

Ramírez Rivera, Hugo Rodolfo. Fuentes para el estudio de la Historia de Chile. Santiago de Chile: UNESCO, Dirección de Bibliotecas, Archivos y Museos, 1984.

Rodríguez, Pedro, Conde de Campomanes. Discurso sobre la educación popular de los artesanos y su fomento. Madrid: Imprenta de Antonio de Sancha, 1775.

Serrano, Sol. "La Revolución Francesa y la formación nacional de educación en Chile", en Krebs, Ricardo y Gazmuri, Cristián. La Revolución Francesa y Chile, Santiago de Chile: Universitaria, 1990, 247-275.

Vergara Villegas, Ignacio. La enseñanza del dibujo artístico en Chile. 1797-1858, Tesis Doctoral leída en la Universitat Politècnica de València, Departamento de Dibujo, 2009.

Villalobos Rivera, Sergio y otros. Historia de Chile. Santiago de Chile: Editorial Universitaria, 1991. 